\title{
MAXIMAL AND MINIMAL RING TOPOLOGIES
}

\author{
NIEL SHELL ${ }^{1}$
}

\begin{abstract}
An explicit description is given of a nondiscrete ring topology on the field $Q$ of rational numbers which is strictly finer than the locally bounded topology on $Q$ having the ring of integers as a preorder. It is observed that either there exist nonvaluable minimal ring topologies or there exist ring topologies containing no minimal ring topologies.
\end{abstract}

It is shown by example that the strongest nondiscrete non-Archimedean ring topology on the field of rational numbers, which is also maximal among all nondiscrete locally bounded ring topologies on the rationals, is not maximal among all nondiscrete ring topologies on the rationals. The existence of minimal and maximal ring topologies is discussed briefly.

The term maximal (minimal) topology on a ring will mean a topology that is maximal (minimal) in the lattice under set inclusion of all nontrivial, nondiscrete ring topologies on the ring. A ring topology is called non-Archimedean if its uniformity is non-Archimedean (see [5]), i.e., if there exists a base at zero of open additive subgroups. If $A$ and $B$ are subsets of a ring, $A-B=\{a-b \mid a \in A, b \in B\}$.

THEOREM 1. Every nontrivial, nondiscrete ring topology is contained in a maximal topology.

Proof. If $T$ is a totally ordered collection of nondiscrete ring topologies stronger than a given ring topology, then $\Re=\cup_{\mathcal{F} \in T} \Re_{\mathcal{F}}$, where $\Re_{\sigma}$ is the $\mathcal{T}$-neighborhood filter at zero, is the neighborhood filter at zero of a ring topology stronger than any topology in $T$. The topology determined by $\Re$ is nondiscrete because $\{0\} \notin \Re_{\sigma}$ for any $\mathcal{T} \in T$. The theorem now follows by Zorn's lemma.

COROLLARY 1.1. There are maximal topologies on every infinite field.

Proof. Theorem 5.2 of [3] states that there are nontrivial, nondiscrete ring topologies on every infinite field.

The locally bounded topology $\mathscr{T}_{Z}$ which has the integers $Z$ as a preorder is

Received by the editors May 20, 1977.

AMS (MOS) subject classifications (1970). Primary 12J99; Secondary 06A20, 13J99, 54A10, $54 \mathrm{E} 15$.

Key words and phrases. Field topology, non-Archimedean ring topology, non-Archimedean uniformity, minimal ring topology, maximal ring topology, nonideal topology, sigma-bounded ring topology, lattice of ring topologies.

${ }^{1}$ The author was formerly known as Niel Shilkret. 
the unique strongest non-Archimedean topology on the rational numbers $Q$ : all nonzero subgroups of $Z$ are $\mathcal{T}_{Z}$-open; hence, all nonzero subgroups of $Q$ are $\mathcal{T}_{Z}$-open. The topology $\mathcal{T}_{Z}$ is also a maximal nondiscrete locally bounded ring topology (see [4]). It is natural to ask if $\mathcal{T}_{Z}$ is a maximal topology.

LEMMA 2.1. If $T$ is a neighborhood base at zero of a ring topology on $Z$, then $\left\{m V \mid m \in Z^{+}, V \in \mathscr{B}\right\}$ is a subbase at zero of a (possibly discrete) ring topology $\mathcal{T}$ on $Q ; \mathcal{T}_{Z} \subset \mathcal{T}$.

Proof. If $U_{i}-U_{i} \subset V_{i}$, then

$$
\left(\cap m_{i} U_{i}\right)-\left(\cap m_{i} U_{i}\right) \subset\left(\cap m_{i} V_{i}\right)
$$

If $a U_{i} \subset V_{i}$, then

$$
\frac{a}{b}\left(\cap b m_{i} U_{i}\right) \subset\left(\cap m_{i} V_{i}\right) .
$$

If $W_{i}+\cdots+W_{i} \subset V_{i}$, where there are $m_{i}$ summands $W_{i}$ on the left side of the containment, and $U_{i}^{2} \subset W_{i}$, then

$$
\begin{aligned}
\left(\bigcap m_{i} U_{i}\right)^{2} \subset & {\left[\cap m_{i}\left(m_{i} W_{i}\right)\right] } \\
\subset & {\left[\cap m_{i}\left(W_{i}+\cdots+W_{i}\right)\right] \subset\left(\bigcap m_{i} V_{i}\right) . }
\end{aligned}
$$

Since $Z$ is a $\mathcal{T}$-neighborhood of zero, so are the sets in the $\mathcal{T}_{Z}$-base $\{n Z\}_{n \in Z^{+}} ;$therefore, $\sigma_{Z} \subset \mathcal{T}$.

THEOREM 2. Let $\left\{a_{1}, a_{2}, \ldots\right\}$ be a sequence of integers greater than two such that, for each $n \in Z^{+}$,

(i) $n ! \mid a_{n}$,

(ii) $a_{n} \mid a_{n+1}$,

(iii) $a_{n+1}>\left(2 n a_{n}+1\right)^{2^{n+1}}$.If

If

$$
\begin{aligned}
V_{r, n} & =\left\{x \in Z \mid x \equiv a\left(2 a_{n} Z\right) \text { for some } a \cdot \ni \cdot|a|<\sqrt[2 r]{2 a_{n}-1}\right\} \\
V_{r} & =\bigcap_{n=1}^{\infty} V_{r, n},
\end{aligned}
$$

then $\mathcal{S}=\left\{m V_{r} \mid m, r \in Z^{+}\right\}$is a neighborhood subbase at zero of a nondiscrete ring topology $\mathcal{T}$ on $Q$ which is strictly finer than $\mathcal{T}_{Z}$.

Proof. Lemma 2.1 will be applied to show that $\mathcal{S}$ determines a ring topology on $Q$ at least as fine as $\widetilde{T}_{z}$. Clearly $\left\{V_{r}\right\}$ is a decreasing sequence of symmetric sets containing zero. Let $c=\left(2 a_{n}\right)^{2^{r}}$. Suppose $x, y \in V_{r+1}$ and

$$
\begin{aligned}
& x \equiv a\left(2 a_{n} Z\right), \quad|a|<\sqrt{c}-1, \\
& y \equiv b\left(2 a_{n} Z\right), \quad|b|<\sqrt{c}-1 .
\end{aligned}
$$

If $c<4$, then $a=b=0$, which implies $|a+b|<c-1$; if $c \geqslant 4$, then $|a+b|<2 \sqrt{c}-2<2 \sqrt{c}-1 \leqslant c-1$. Therefore, $x+y \in V_{r}$. Also, 


$$
|a b|<(\sqrt{c}-1)^{2}=c-(2 \sqrt{c}-1)<c-1 .
$$

Therefore $x y \in V_{r}$. From the containments $V_{r+1}+V_{r+1} \subset V_{r}$ and $V_{r+1}^{2} \subset$ $V_{r}$, which have just been established, it follows that, for any $k \in Z$,

$$
k V_{r+|k|} \subset V_{r+|k|}+\cdots+V_{r+|k|} \subset V_{r},
$$

where there are $2^{|k|}$ summands $V_{r+|k|}$ in the middle member of this chain of containments. It now follows that $\left\{V_{r}\right\}$ is the neighborhood base at zero of a ring topology on $Z$.

Next it is shown that $\mathcal{T} \neq \mathcal{T}_{Z}$ by showing that only the zero ideal of $Z$ is contained in $V_{1}$. Since $2 \leqslant a_{n}$, for all $n, \sqrt{2 a_{n}} \leqslant a_{n}$. Since $\pm a_{n}$ are the representatives of $a_{n}$ modulo $2 a_{n} Z$ with smallest absolute value, $a_{n} \notin V_{n, 1} \supset$ $V_{1}$. But, since $n ! \mid a_{n}$, the sequence $\left\{a_{n}\right\}$ is eventually in every nonzero ideal of $Z$.

Finally it is shown that $\mathcal{T}$ is not discrete by observing that

$$
2 a_{R} \prod_{i=1}^{t} m_{i} \in \bigcap_{i=1}^{t} m_{i} V_{r},
$$

for $R \geqslant \max \left(r, \Pi_{i=1}^{t} m_{i}\right)$. Let $x=2 a_{R} \Pi m_{i}$. Since $a_{n} \mid x / m_{i}$ if $n \leqslant R, x / m \in$ $V_{r, n}$ for $n \leqslant R$. If $n>R$, then

$$
0 \leqslant \frac{x}{m_{i}} \leqslant 2 R a_{R}<\sqrt[2^{R+1}]{a_{R+1}}-1 \leqslant \sqrt[2 r]{a_{n}}-1 .
$$

where the third inequality is a consequence of property (iii) of $\left\{a_{r}\right\}$.

The proof of Theorem 2 contains an explicit description of a nondiscrete ring topology on $Z$ which is stronger than the topology with all nonzero ideals as a basis at zero. The existence of "nonideal" topologies was established in [2].

A subset of a topological ring will be called $\sigma$-bounded if it can be written as a countable union of bounded sets; a ring topology will be called $\sigma$-bounded if the entire ring is a $\sigma$-bounded set. Since finite sets are bounded in any ring topology, all ring topologies on a countable ring are $\sigma$-bounded.

THEOREM 3. Any nontrivial $\sigma$-bounded ring topology on a ring $R$ can be weakened to a nontrivial first countable ring topology.

Proof. Suppose $R=\cup_{n=1}^{\infty} B_{n}$, where $\left\{B_{n}\right\}$ is an increasing sequence of sets bounded in topology $\sigma$. Let $V_{1}$ be any neighborhood of zero distinct from $R$. Inductively, choose a sequence $\left\{V_{n}\right\}$ of symmetric $\sigma_{\text {-open neigh- }}$ borhoods of zero such that $V_{n+1}+V_{n+1} \subset V_{n} ; V_{n+1}^{2} \subset V_{n} ; B_{n} V_{n+1} \subset V_{n}$ and $V_{n+1} B_{n} \subset V_{n}$. Then $\left\{V_{n}\right\}$ is a neighborhood base at zero for a first countable ring topology contained in $\mathcal{T}$.

COROLlaRY 3.1. All nontrivial $\sigma$-bounded minimal topologies on a ring are first countable. In particular, all minimal topologies on a countable field are first countable.

There are ring topologies on a simple transcendental extension $C(t)$ of the 
complex field $C$ which induce the usual topology on $C$; examples of such topologies are given in [6]. Let $\sigma$ be such a topology. Suppose there existed a valuation || on $C(t)$ such that the associated value topology $\sigma_{\mid}$, were contained in $\mathcal{T}_{\text {. Then }} \widetilde{T}_{11}$ would induce a ring topology on $C$ which would be contained in the usual topology on $C$. But the topology induced on $C$ by $\mathscr{T}_{\mid 1}$ would be the valuable (hence nontrivial) topology of the restriction of || ; and, furthermore, the usual topology on $C$ is minimal. Therefore, || would induce the usual topology on $C$. But this is a contradiction, since no valuation on $C(t)$ can be Archimedean on $C$. Hence, a ring topology on $C(t)$ which induces the usual topology on $C$ does not contain a valuable topology. Thus, (at least) one of two interesting situations must occur: either there exist nonvaluable minimal topologies or there exist topologies which contain no minimal topologies.

\section{REFERENCES}

1. J. Heine and S. Warner, Ring topologies on the quotient field of a Dedekind domain, Duke Math. J. 40 (1973), 473-486.

2. L. A. Hinrichs, Integer topologies, Proc. Amer. Math. Soc. 15 (1964), 991-995.

3. J. O. Kiltinen, Inductive ring topologies, Trans. Amer. Math. Soc. 134 (1968), 149-169.

4. M. E. Shanks and S. Warner, Topologies on the rational field, Bull. Amer. Math. Soc. 79 (1973), 1281-1282.

5. N. Shilkret, Non-Archimedean orthogonality, Arch. Math. 27 (1976), 67-78.

6. J. H. Williamson, On topologizing the field $C(t)$, Proc. Amer. Math. Soc. 5 (1954), 729-734.

Department of Mathematics, City College of New York (CUNY), New York, New YORK 10031 